\title{
The Effect of Metformin on Survival Outcomes of Non-Metastatic Breast Cancer Patients with Type 2 Diabetes
}

\author{
Bita Behrouzi ${ }^{1}$, Mohammad Zokaasadi², Mohhamad Ali Mohagheghi ${ }^{3}$, Amir \\ Hosein Emami ${ }^{4}$, Sanambar Sadighi ${ }^{4 *}$
}

\begin{abstract}
Background: There are still inconsistencies about the role of metformin on breast cancer. This study was designed to assess metformin's effect on the prognosis of female breast cancer patients with type II diabetes. Methods: The present research was carried out as a retrospective cohort study between 2003 and 2014. Breast cancer patients with pre-existing type II diabetes mellitus were included. Overall survival (OS) and relapse-free survival (RFS) were measured as the main endpoints. Kaplan-Meier estimate was used to calculate survival rates. Results: 217 patients were included with a mean age of $53.32 \pm 11.10$ years. $148(68.2 \%)$ patients were prescribed metformin and $69(31.8 \%)$ took other antidiabetic drugs (non-metformin group). Five-year OS and RFS rates for all patients were $82.5 \%$ (95\% CI: 76.0\%-87.4\%) and $71.1 \%$ (95\% CI: 64.2\%-77.0\%) respectively. Log-rank test showed that the metformin group had a significant advantage over the non-metformin group in terms of both OS and RFS rates $(\mathrm{P}<0.001$ for both). Five-year OS and RFS rates for metformin group were $91.9 \%$ (95\% CI: 85.4\%-95.6\%) and 82.8\% (95\%CI: $75.5 \%-88.2 \%)$ respectively; the same rates for non-metformin group were 59.1\% (95\% CI: 43.9\%-71.5\%) and 39.3\% (95\%CI: 25.1\%-53.1\%) (P<0.001 for both). Results of proportional hazards model, after adjustment for body mass index, age, and tumor stage, depicted an independent prognostic value of metformin use with multivariate hazard ratio of 0.15 (95\% CI: 0.07-0.32) for OS and 0.23 (0.14-0.40) for RFS compared to non-metformin group ( $\mathrm{P}<0.001$ for both). Conclusion: This study indicated that using metformin for diabetic breast cancer patients is associated with favorable results regarding recurrence and survival rates.
\end{abstract}

Keywords: Breast neoplasms- survival- metformin

Asian Pac J Cancer Prev, 22 (2), 611-616

\section{Introduction}

Breast cancer is the most common cancer in females around the globe causing a substantial number of deaths annually (Siegel et al., 2019). Based on the Global Cancer Observatory report, in 2018, breast cancer occupied the first rank as the most frequent type of cancer and the second most common cause of cancer death among the whole population (Bray et al., 2018). It is also the most frequent cancer of Iranian women with an age-standardized ratio of 33.2 breast cancer cases per 100,000 (Nafissi et al., 2018). Diabetes mellitus (DM) is a major health problem in Iran and globally, with its prevalence in the Iranian adult population reaching 11.4\% based on recent investigations (Esteghamati et al., 2017). Notably, statistics on DM show a growing trend compared to the previous decade (Esteghamati et al., 2017). There is evidence supporting that DM patients are at increased risk of developing more invasive forms of cancer (Gong et al., 2016) and that diabetic breast cancer patients have a worse outcome compared to non-diabetics (Peairs et al., 2011; Behrouzi et al., 2017). Metformin, a biguanide antidiabetic agent used as a first-line therapy for type $2 \mathrm{DM}$ particularly in overweight and obese patients, has recently come back into the realm of translational and clinical oncology research due to its potential effects on reduction of cancer incidence (Chlebowski et al., 2012) as well as its synergistic effects on cancer treatment (Chuang et al., 2018; Yu et al., 2019). This impact has been examined in a vast majority of solid tumors (e.g. colorectal, pancreas and lung) (Dowling et al., 2011; Ramjeesingh et al., 2016; Xu et al., 2018; Zhang et al., 2018; He et al., 2019). Both in vitro and in vivo studies on the role of metformin on breast cancer have been performed but have not reached a consistent result. The metformin effect on breast cancer still remains controversial (Zakikhani et al., 2006;

${ }^{1}$ Geisel School of Medicine at Dartmouth, Hanover, NH, USA. ${ }^{2}$ Hematology, Oncology and Stem Cell Transplantation Research Center, Tehran University of Medical Sciences, Tehran, Iran. ${ }^{3}$ The Cancer Research Center of the Cancer Institute, Tehran University of Medical Sciences, Tehran, Iran. ${ }^{4}$ Department of Hematology-Oncology, Cancer Institute of Iran, Tehran University of Medical Sciences, Tehran, Iran.*For Correspondence: ssadighi@tums.ac.ir 
Vazquez-Martin et al., 2011; Lega et al., 2013; Oppong et al., 2014). Therefore, we conducted a retrospective study to evaluate the effect of metformin in a cohort of diabetic breast cancer patients.

\section{Materials and Methods}

From July 2003 to February 2014, diabetic patients undergoing treatment for breast cancer at the Cancer Institute of Iran were chosen for this study. Inclusion criteria were stages I to III breast cancer at the time of diagnosis and pre-existing type II DM. Exclusion criteria were breast cancer with distant metastasis, gestational DM, steroid-induced DM, type I DM and male breast cancer. All patients received chemotherapy and/or hormonal therapy. The patients were followed until death or December 2016. Since some limitation in research budgets we were not able to prolong the follow up period beyond the end of 2016. DM type II diagnosis was confirmed by blood glucose testing and self-report. The antidiabetic medication was extracted from the medical files and the patients were categorized into one of the two groups: metformin users, comprising those who had took at least 1000 milligrams of metformin during the follow up period and non-metformin group, including those used other antidiabetic drugs. The study protocol was approved by Ethics Committee of our institution.

TNM staging based on the American Joint Committee on Cancer's 2020 manual was used for staging the tumors. Hormonal status of the samples for estrogen and progesterone receptors (ER and PR) were tested by immunohistochemistry (IHC). A sample was confirmed as HER2 positive once one of the following criteria were met: three positive overexpression in IHC was seen or Fluorescent in Situ Hybridization (FISH) amplification was observed. Tumor subtypes were categorized based on hormonal and HER2 status: luminal A (ER positive and/or PR positive, HER2 negative), luminal B (ER positive and/or PR positive, HER2 positive), HER2 enhanced type (ER negative, PR negative, HER2 positive), and triple-negative (ER negative, PR negative, HER2 negative). All specimens were rechecked for the purpose of the research.

Differences were tested using the Chi-square test for categorical variables and the Independent Samples t-Test for continuous variables. Overall survival (OS) was defined as the time from pathologically confirmed diagnosis to all-cause death or end of the follow-up time. Recurrence-free survival (RFS) time was defined as the time from diagnosis to the first local, regional or distant recurrence of the disease. Kaplan-Meier estimate was used to calculate the survival rates. The difference between metformin and non-metformin groups was compared by the log-rank method. Cox regression analysis (uni- and multivariate analysis) was used to assess the effect of variables on survival outcomes. Tests were 2-sided and $\mathrm{P}$-values of less than 0.05 were considered significant. Analyses were performed by $\mathrm{R}$ software for windows version 3.5.1 (survival package) and IBM SPSS statistics version 23 .

\section{Results}

A total of 217 female diabetic patients were included in the study. Mean age at diagnosis was $53.32 \pm 11.10$ years. Amongst patients, 148 were treated by metformin (68.2\%) and 69 (31.8\%) used other antidiabetic drugs. There was no significant difference between metformin and non-metformin groups regarding age, weight, height, body mass index (BMI), hormone receptor status, tumor subtype, chemotherapy protocol and tumor stage. Basic characteristics of the studied patients were summarized in Table 1.

During a median follow-up time of 60 months (ranging from 1 to 160 months), a total of 37 deaths occurred, of which $11(29.73 \%)$ were in the metformin group and the remaining $26(70.27 \%$ ) were in the non-metformin group $(\mathrm{P}<0.001)$. Mortality rates were $7.43 \%$ for the metformin group and $37.68 \%$ for the non-metformin group. KaplanMeier estimate for all patients showed 1- and 5-year OS rates of $97.7 \%$ (95\% CI: $94.5 \%-99.0 \%)$ and $82.5 \%$
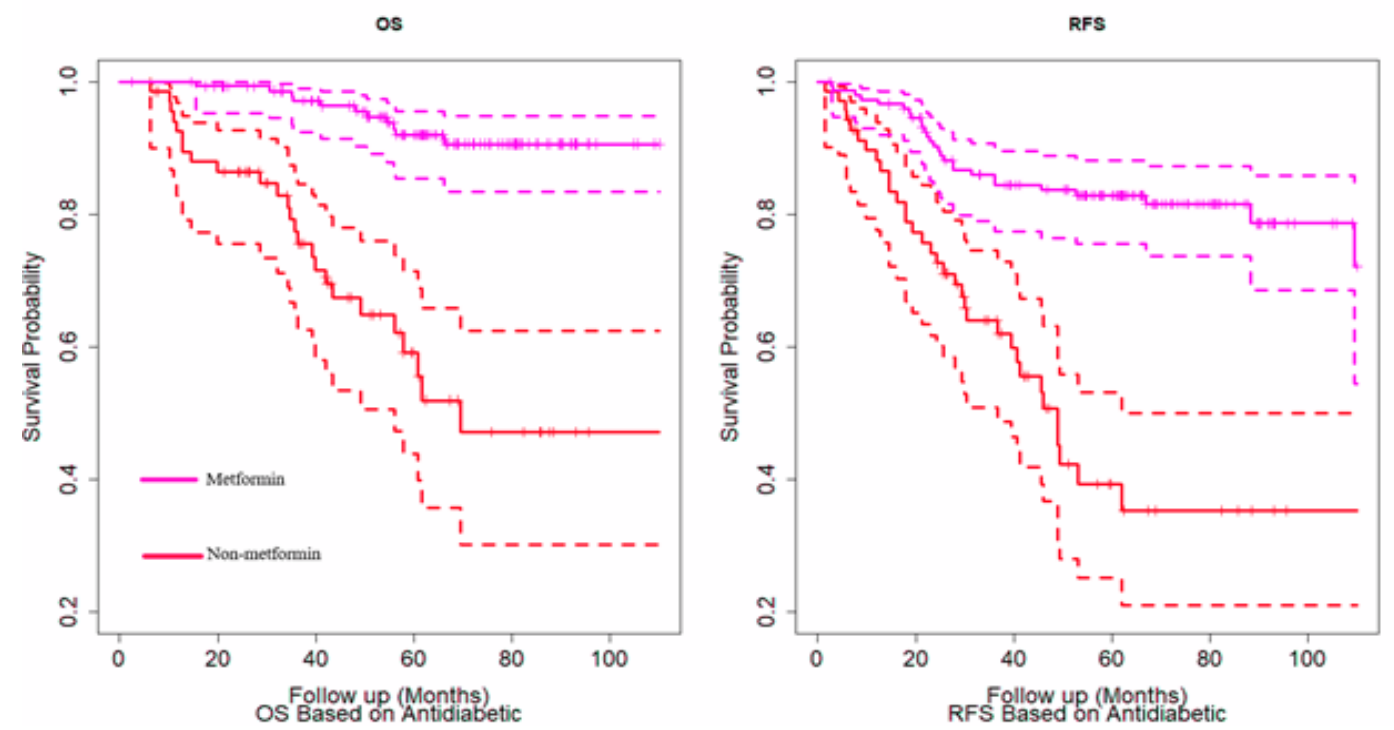

Figure 1. OS (Overall Survival) and RFS (Recurrence-Free Survival) Curves (Solid Line) for Metformin and Non-Metformin Groups with Corresponding 95\% Confidence Intervals (Dashed Lines) 
Table 1. Basic Characteristics of Studied Population

\begin{tabular}{|c|c|c|c|c|}
\hline Variable & Total $(\mathrm{N}=217)$ & Non-metformin $(\mathrm{N}=69)$ & Metformin $(\mathrm{N}=148)$ & $\mathrm{P}$ \\
\hline \multicolumn{5}{|l|}{ Age at diagnosis, years } \\
\hline Mean \pm SD & $53.32 \pm 11.10$ & $55.22 \pm 11.20$ & $52.44 \pm 10.98$ & 0.09 \\
\hline \multicolumn{5}{|l|}{ Body weight, $\mathrm{Kg}$} \\
\hline Mean \pm SD & $74.10 \pm 13.78$ & $75.21 \pm 14.04$ & $73.59 \pm 13.67$ & 0.43 \\
\hline \multicolumn{5}{|l|}{ Height, $\mathrm{cm}$} \\
\hline Mean \pm SD & $154.45 \pm 6.63$ & $153.66 \pm 6.31$ & $154.81 \pm 6.76$ & 0.24 \\
\hline \multicolumn{5}{|l|}{ Body Mass Index, kg/m² } \\
\hline Mean \pm SD & $31.13 \pm 5.80$ & $31.96 \pm 6.29$ & $30.75 \pm 5.54$ & 0.16 \\
\hline$<35 \mathrm{n}(\%)$ & $167(79.15 \%)$ & $48(71.64 \%)$ & $119(82.64 \%)$ & 0.07 \\
\hline$\geq 35 \mathrm{n}(\%)$ & $44(20.85 \%)$ & $19(28.36 \%)$ & $25(17.36 \%)$ & \\
\hline \multicolumn{5}{|l|}{ ER } \\
\hline Negative n (\%) & $65(30.1 \%)$ & $24(35.29 \%)$ & $41(27.70 \%)$ & 0.27 \\
\hline Positive $n(\%)$ & $151(69.9 \%)$ & $44(64.71 \%)$ & $107(72.30 \%)$ & \\
\hline \multicolumn{5}{|l|}{ PR } \\
\hline Negative n (\%) & $79(36.57 \%)$ & $29(42.65 \%)$ & $50(33.78 \%)$ & 0.23 \\
\hline Positive n (\%) & $137(63.43 \%)$ & $39(57.35 \%)$ & $98(66.22 \%)$ & \\
\hline \multicolumn{5}{|l|}{ HER2 } \\
\hline Negative n (\%) & $170(79.07 \%)$ & $56(82.35 \%)$ & $115(77.70 \%)$ & 0.48 \\
\hline Positive n (\%) & $45(20.93 \%)$ & $12(17.65 \%)$ & $33(22.30 \%)$ & \\
\hline \multicolumn{5}{|l|}{ Subtype } \\
\hline Luminal-A n (\%) & $131(60.93 \%)$ & $40(58.82 \%)$ & $91(61.90 \%)$ & 0.45 \\
\hline Luminal-B n (\%) & $19(8.84 \%)$ & $4(5.88 \%)$ & $15(10.20 \%)$ & \\
\hline Triple-Negative n $(\%)$ & $39(18.14 \%)$ & $16(23.53 \%)$ & $23(15.65 \%)$ & \\
\hline HER2type n (\%) & $26(12.09 \%)$ & $8(11.76 \%)$ & $18(12.24 \%)$ & \\
\hline \multicolumn{5}{|l|}{ Chemotherapy } \\
\hline Adjuvant n (\%) & $196(90.32 \%)$ & $59(85.51 \%)$ & $137(92.57 \%)$ & 0.14 \\
\hline Neo-adjuvant n (\%) & $21(9.68 \%)$ & $10(14.49 \%)$ & $11(7.43 \%)$ & \\
\hline \multicolumn{5}{|l|}{ TNM stage } \\
\hline $\operatorname{In}(\%)$ & $35(16.59 \%)$ & $9(13.64 \%)$ & $26(17.93 \%)$ & 0.66 \\
\hline IIA n(\%) & $55(26.07 \%)$ & $14(21.21 \%)$ & $41(28.28 \%)$ & \\
\hline IIB n(\%) & $46(21.80 \%)$ & $16(24.24 \%)$ & $30(20.69 \%)$ & \\
\hline IIIA n(\%) & $44(20.85 \%)$ & $14(21.21 \%)$ & $30(20.69 \%)$ & \\
\hline IIIB n(\%) & $14(6.64 \%)$ & $6(9.09 \%)$ & $8(5.52 \%)$ & \\
\hline IIIC n(\%) & $17(8.06 \%)$ & $7(10.61 \%)$ & $10(6.90 \%)$ & \\
\hline
\end{tabular}

ER, estrogen receptor; PR, progesterone receptor

(95\% CI: $76.0 \%-87.4 \%$ ) respectively. The number of recurrences was significantly lower in the metformin group with 28 recurrences in the metformin group compared to 35 recurrences in the non-metformin group $(\mathrm{P}<0.001)$. Relapse rates were $18.92 \%$ for the metformin group and $50.72 \%$ for the non-metformin group. RFS rates for the 1 st and 5 th years were $94.4 \%$ (95\% CI: 90.3\%$96.8 \%$ ) and $71.1 \%$ (95\% CI: $64.2 \%-77.0 \%)$ respectively for all patients. Log-rank test revealed that the metformin group had significantly superior OS and RFS rates. Results of the log-rank tests are summarized in Table 2.

5 -year OS rates in the metformin and the non-metformin group were $91.9 \%(85.4 \%-95.6 \%)$ and $59.1 \%$ (43.9\%-71.5\%) while the RFS rates were $82.8 \%$ (75.5\%-88.2\%) and 39.3\% (25.1\%-53.1\%) respectively $(\mathrm{P}<0.001$ for both). These significant differences were shown in Figure 1.

The proportional hazards model presented three prognostic factors affecting OS and RFS: tumor stage, $\mathrm{BMI}$ and use of metformin. After adjusting for age, tumor stage and BMI, metformin usage resulted in $85 \%$ lower risk of death and $77 \%$ lower risk of relapse. Hazard ratio was 0.15 (95\% CI: 0.07-0.32; $\mathrm{P}<0.001)$ for OS and 0.23 (0.14-0.40; $\mathrm{P}<0.001)$ for RFS respectively compared to the non-metformin group. Results of the Cox proportional hazards model were summarized in Table 3.

\section{Discussion}

This study was designed to assess the role of metformin on the treatment of female breast cancer patients with type II DM. Our results showed a significant 
Table 2. Differences of OS and RFS Based on Prognostic Factors

\begin{tabular}{|c|c|c|c|c|c|}
\hline Covariate & & 5-year OS $(95 \% \mathrm{CI})$ & $\mathrm{P}$ & 5-year RFS $(95 \% \mathrm{CI})$ & $\mathrm{P}$ \\
\hline \multirow[t]{2}{*}{ Antidiabetic } & Metformin & $91.9(85.4-95.6)$ & $<0.001$ & $82.8(75.5-88.2)$ & $<0.001$ \\
\hline & Non-metformin & $59.1(43.9-71.5)$ & & $39.3(25.1-53.1)$ & \\
\hline \multirow[t]{4}{*}{ Subtype } & Luminal A & $83.6(75.1-89.4)$ & A 0.8 & $71.9(62.8-79.1)$ & 0.9 \\
\hline & Luminal B & $77.9(35.4-94.2)$ & & $71.5(43.8-87.2)$ & \\
\hline & HER2 type & $79.3(57.1-90.9)$ & & $77.1(52.4-90.1)$ & \\
\hline & TNBC & $80.6(63.6-90.3)$ & & $67.2(49.4-79.9)$ & \\
\hline \multirow[t]{2}{*}{ Age, years } & $>50$ & $79.3(70.0-86.0)$ & 0.3 & $77.6(66.3-85.4)$ & 0.3 \\
\hline & $\leq 50$ & $86.6(76.5-92.6)$ & & $66.6(57.2-74.4)$ & \\
\hline \multirow[t]{2}{*}{ BMI, kg/m² } & $\geq 35$ & $62.9(45.7-76.0)$ & $<0.001$ & $53.7(37.2-67.7)$ & 0.005 \\
\hline & $<35$ & $88.2(81.4-92.7)$ & & $76.2(68.4-82.2)$ & \\
\hline \multirow[t]{6}{*}{ TNM Stage } & I & $96.4(77.2-99.5)$ & $<0.001$ & 97.1 (80.9-99.6) & $<0.001$ \\
\hline & IIA & $96.3(85.8-99.1)$ & & $86.5(73.7-93.3)$ & \\
\hline & IIB & $76.0(58.5-86.9)$ & & $69.9(52.8-81.8)$ & \\
\hline & IIIA & $75.4(57.8-86.4)$ & & $60.8(44.0-74.0)$ & \\
\hline & IIIB & $60.6(29.4-81.4)$ & & $39.3(14.5-63.6)$ & \\
\hline & IIIC & $61.9(33.9-80.8)$ & & $43.9(19.9-65.7)$ & \\
\hline
\end{tabular}

TNBC, triple negative breast cancer; BMI, body mass index; RFS, recurrence-free survival; OS, overall survival; CI, confidence interval.

prognostic value of metformin compared to other antidiabetic agents in breast cancer patients which is in line with some previous references; the study of $\mathrm{He}$ et al at MD Anderson Cancer Center on 1,983 patients with the HER2 positive subtype of breast cancer showed that DM type II was an independent prognostic factor of poor OS in stage II or more of the disease, after adjustment for age, BMI, hormonal receptor status, nuclear grade, and stage. Another important finding of the mentioned study was that among breast cancer patients with DM type II, usage of metformin and thiazolidinediones were associated with improved OS (He et al., 2011). However, the study of Bayraktar et al from the same center did not show any significant advantage for diabetic metformin users in the adjuvant setting (Bayraktar et al., 2012); that subset of patients had poor prognostic factors of being obese, having TNBC pathologic subtype, or being black.

A population-based study on older breast cancer patients (aged $\geq 66$ years) was the first research to evaluate the time-dependent effects of metformin; even though a 5 -year cumulative metformin usage led to $38 \%$ decrease in cancer-specific mortality, the HR was not significant (Lega et al., 2013). The authors discussed the limited proportion of patients with long follow-up time as the cause for lack

Table 3. Results of Univariate and Multivariate Analyses

\begin{tabular}{|c|c|c|c|c|c|c|c|c|}
\hline \multirow[t]{3}{*}{ Covariate } & \multicolumn{4}{|c|}{ OS } & \multicolumn{4}{|c|}{ RFS } \\
\hline & \multicolumn{2}{|l|}{ Univariate } & \multicolumn{2}{|l|}{ Multivariate } & \multicolumn{2}{|l|}{ Univariate } & \multicolumn{2}{|l|}{ Multivariate } \\
\hline & HR (CI 95\%) & $\mathrm{P}$ & HR (CI 95\%) & $\mathrm{P}$ & HR (CI 95\%) & $\mathrm{P}$ & HR (CI 95\%) & $\mathrm{P}$ \\
\hline Age & $1.03(1.00-1.06)$ & 0.09 & $1.01(0.97-1.05)$ & 0.58 & $1.01(0.99-1.04)$ & 0.28 & & \\
\hline \multicolumn{9}{|l|}{ Subtype } \\
\hline Luminal A & 1 & 0.83 & & & 1 & 0.92 & & \\
\hline Luminal B & $0.72(0.17-3.07)$ & 0.66 & & & $1.00(0.39-2.53)$ & 0.99 & & \\
\hline TNBC & $1.14(0.49-2.66)$ & 0.76 & & & $1.15(0.60-2.19)$ & 0.68 & & \\
\hline HER2 type & $1.43(0.54-3.78)$ & 0.47 & & & $0.79(0.31-2.00)$ & 0.61 & & \\
\hline \multicolumn{9}{|l|}{ TNM Stage } \\
\hline I & 1 & 0.001 & 1 & 0.003 & 1 & $<0.001$ & 1 & $<0.001$ \\
\hline IIA & $1.21(0.11-13.35)$ & 0.88 & $1.53(0.14-17.05)$ & 0.73 & $5.79(0.73-45.69)$ & 0.1 & $5.88(0.73-47.25)$ & 0.1 \\
\hline IIB & $8.92(1.15-69.25)$ & 0.04 & $8.12(1.05-63.12)$ & 0.045 & $10.91(1.43-83.44)$ & 0.02 & $10.96(1.43-84.06)$ & 0.02 \\
\hline IIIA & $8.45(1.09-65.65)$ & 0.04 & $7.29(0.94-56.89)$ & 0.06 & $15.95(2.13-119.70)$ & 0.007 & $16.75(2.22-126.13)$ & 0.006 \\
\hline IIIB & $21.24(2.48-182.21)$ & 0.005 & $30.18(3.26-279.79)$ & 0.003 & $39.24(4.89-314.64)$ & 0.001 & $36.94(4.52-301.78)$ & 0.001 \\
\hline IIIC & $20.65(2.53-168.53)$ & 0.005 & $13.88(1.70-113.74)$ & 0.01 & $30.68(3.88-242.78)$ & 0.001 & $30.37(3.82-241.43)$ & 0.001 \\
\hline $\operatorname{BMI}\left(\mathrm{kg} / \mathrm{m}^{2}\right)$ & $1.10(1.05-1.16)$ & $<0.001$ & $1.08(1.03-1.14)$ & 0.002 & $1.05(1.00-1.09)$ & 0.04 & $1.05(1.01-1.10)$ & 0.02 \\
\hline \multicolumn{9}{|l|}{ Antidiabetic agent } \\
\hline $\begin{array}{l}\text { Metformin (REF: } \\
\text { non-metformin) }\end{array}$ & $0.12(0.06-0.25)$ & $<0.001$ & $0.15(0.07-0.32)$ & $<0.001$ & $0.24(0.14-0.39)$ & $<0.001$ & $0.23(0.14-0.40)$ & $<0.001$ \\
\hline
\end{tabular}

TNBC, triple negative breast cancer; BMI, body mass index; RFS, recurrence-free survival; OS, overall survival; CI, confidence interval; REF, reference. 
of power and non-significant results. Another singlecenter study on 141 patients with type II DM revealed slightly higher OS and RFS rates in metformin users, yet the difference failed to attain the statistical level of significance (Oppong et al., 2014). Considering these controversies and lack of an agreement, some systematic reviews were performed in this field. The study of Xu et al, which analyzed 11 original studies, depicted a substantial role of metformin on overall survival of diabetic patients with breast cancer with HR of 0.35 (95\% CI: 0.15-0.84) (Xu et al., 2015). Recent data from the Women's Health Initiative also confirmed the benefit of metformin in terms of cancer-related mortality in breast cancer patients (Gong et al., 2016). Another meta-analysis, adding together 4 studies with and 4 other studies without significant results, concluded that the metformin effect on all-cause mortality of breast cancer was statistically significant (pooled HR: $0.63, \mathrm{P}<0.001$ ) (Cao et al., 2017). Similarly a bigger review confirmed the positive effect of metformin on OS of breast cancer patients (Yu et al., 2019).

Metformin has also been shown to be effective in malignancies of the gastrointestinal tract, lung, and prostate cancers (He et al., 2019; Chuang et al., 2018; Ramjeesingh et al., 2016; Xu et al., 2018; Zhang et al., 2018). Efficacy of metformin on different solid tumors, as well as its effects on both diabetic and non-diabetic patients, suggests an intertwined nexus of action in different organ systems. There are two main possible pharmacological pathways for metformin anti-cancer effects. The first is the insulin-dependent pathway which relies on insulin lowering function of the drug, thus decreasing proliferative capacity of the tumor (Heckman-Stoddard et al., 2017). The second is the insulin-independent pathway which acts directly on cancer cells (Gonzalez-Angulo and Meric-Bernstam, 2010). A key molecule affected by metformin in both pathways is 5 ' adenosine monophosphate-activated protein kinase (AMPK) (Goodwin et al., 2009), which is a eukaryotic energy sensor (Alimova et al., 2009). Activation of AMPK causes inhibition of the mammalian target of rapamycin (mTOR) complex I, which in turn leads to inhibition of cell proliferation and biosynthesis of necessary components needed for cell growth and division (Martin-Castillo et al., 2010). An in vitro study on TNBC cell lines indicated that metformin has substantial activity on the induction of apoptosis and cell cycle arrest. However, in some experiments, metformin doses were above the therapeutic range. Given the fact that the metformin effects are timeand dose-dependent (Deng et al., 2012), the possibility for clinical application of these findings is limited (Liu et al., 2009).

Another presumed mechanism for metformin action is alteration of the cyclin D and inhibition of cell cycle progression. Along this line, metformin has been shown to be effective in trastuzumab-resistant breast cancer cells (Liu et al., 2011; Vazquez-Martin et al., 2011). Nonetheless, the exact underlying mechanisms by which metformin induces anti-cancer capabilities are not completely known. The role of metformin in earlystage breast cancer was assessed in non-diabetic women in support of the significant effect of metformin on the reduction of tumor proliferation and increase of apoptosis (Niraula et al., 2012). On the other hand, some clinical window of opportunity studies that explored metformin's effects on non-diabetic patients showed a significant reduction of Ki67 marker in accordance with its effect on glucose and insulin levels (Homeostasis Model Assessment score) (Sadighi et al., 2016). A basic research, through an in vitro study, using breast cancer cell lines of four different subtypes (Luminal-A, luminal-B, HER2 type and TNBC) in normoglycemic settings, failed to show added neoadjuvant value for metformin when compared to a taxane agent alone (Sadighi et al., 2014). Results of these two mentioned studies might be considered as another supporting evidence for indirect anti-cancer effects of metformin through interfering with insulin and insulin-like growth factors.

The retrospective nature of the current study and comparatively small sample size are the main limitations to state a more solid conclusion about metformin's potential on improving prognosis of cancer patients. In general, due to the diverse biologic nature of breast cancer and the manifold molecular mechanisms of metformin effect on different metabolic pathways, it seems that more specifically designed studies (e.g. randomized control trials) are needed to reach a more thorough understanding of its role on either diabetic or non-diabetic breast cancer patients. Well-tolerability, relatively low toxicity profile, availability and low-cost are the factors that should be considered for using metformin as adjuvant therapy. Late phase clinical trials are ongoing such as a Phase III Randomized Trial of Metformin vs. Placebo in Early Stage Breast Cancer sponsored by the Canadian Cancer Trials Group. As the clinical trials are the benchmark in medical research, we need to wait to reach a better understanding of metformin's anti-cancer profile.

\section{References}

Alimova IN, Liu B, Fan Z, et al (2009). Metformin inhibits breast cancer cell growth, colony formation and induces cell cycle arrest in vitro. Cell Cycle, 8, 909-15.

Bayraktar S, Hernadez-Aya LF, Lei X, et al (2012). Effect of metformin on survival outcomes in diabetic patients with triple receptor-negative breast cancer. Cancer, 118, 1202-11.

Behrouzi B, Mohagheghi MA, Sadighi S (2017). Demographic characteristics, survival and prognostic factors of early breast cancer patients with type 2 diabetes mellitus: A HospitalBased Cohort Study. Asian Pac J Cancer Prev, 18, 2485-91.

Bray F, Ferlay J, Soerjomataram I, et al (2018). Global cancer statistics 2018: GLOBOCAN estimates of incidence and mortality worldwide for 36 cancers in 185 countries. $C A$ Cancer J Clin, 68, 394-424.

Cao X, Wu Y, Wang J, et al (2017). The effect of metformin on mortality among diabetic cancer patients: A Systematic Review and Meta-analysis. JNCI Cancer Spectr, 1.

Chlebowski RT, McTiernan A, Wactawski-Wende J, et al (2012). Diabetes, metformin, and breast cancer in postmenopausal women. J Clin Oncol, 30, 2844-52.

Chuang M-C, Yang Y-H, Tsai Y-H, et al (2018). Survival benefit associated with metformin use in inoperable non-small cell lung cancer patients with diabetes: A population-based retrospective cohort study. PLoS One, 13, e0191129.

Deng X-S, Wang S, Deng A, et al (2012). Metformin targets Stat3 
to inhibit cell growth and induce apoptosis in triple-negative breast cancers. Cell Cycle, 11, 367-76.

Dowling RJO, Goodwin PJ, Stambolic V (2011). Understanding the benefit of metformin use in cancer treatment. $B M C$ Med, 9, 33 .

Esteghamati A, Larijani B, Aghajani MH, et al (2017). Diabetes in Iran: Prospective analysis from first nationwide diabetes report of national program for prevention and control of diabetes (NPPCD-2016). Sci Rep, 7, 13461.

Gong Z, Aragaki AK, Chlebowski RT, et al (2016). Diabetes, metformin and incidence of and death from invasive cancer in postmenopausal women: Results from the women's health initiative. Int J Cancer, 138, 1915-27.

Gonzalez-Angulo AM, Meric-Bernstam F (2010). Metformin: a therapeutic opportunity in breast cancer. Clin Cancer Res, 16, 1695-700.

Goodwin PJ, Ligibel JA, Stambolic V (2009). Metformin in breast cancer: time for action. J Clin Oncol, 27, 3271-3.

$\mathrm{He} \mathrm{K}, \mathrm{Hu} \mathrm{H}$, Ye S, et al (2019). The effect of metformin therapy on incidence and prognosis in prostate cancer: A systematic review and meta-analysis. Sci Rep, 9, 2218.

He X, Esteva FJ, Ensor J, et al (2011). Metformin and thiazolidinediones are associated with improved breast cancer-specific survival of diabetic women with HER2+ breast cancer. Ann Oncol, 23, 1771-80.

Heckman-Stoddard BM, DeCensi A, Sahasrabuddhe VV, et al (2017). Repurposing metformin for the prevention of cancer and cancer recurrence. Diabetologia, 60, 1639-47.

Lega IC, Austin PC, Gruneir A, et al (2013). Association Between Metformin Therapy and Mortality After Breast Cancer. A population-based study. Diabetes Care, 36, 3018-26.

Liu B, Fan Z, Edgerton SM, et al (2009). Metformin induces unique biological and molecular responses in triple negative breast cancer cells. Cell Cycle, 8, 2031-40.

Liu B, Fan Z, Edgerton SM, et al (2011). Potent anti-proliferative effects of metformin on trastuzumab-resistant breast cancer cells via inhibition of erbB2/IGF-1 receptor interactions. Cell Cycle, 10, 2959-66.

Martin-Castillo B, Vazquez-Martin A, Oliveras-Ferraros C, et al (2010). Metformin and cancer: Doses, mechanisms and the dandelion and hormetic phenomena. Cell Cycle, 9, 1057-64.

Nafissi N, Khayamzadeh M, Zeinali Z, et al (2018). Epidemiology and histopathology of breast cancer in Iran versus other Middle Eastern countries. Middle East J Cancer, 9, 243-51.

Niraula S, Dowling RJ, Ennis M, et al (2012). Metformin in early breast cancer: a prospective window of opportunity neoadjuvant study. Breast Cancer Res Treat, 135, 821-30.

Oppong BA, Pharmer LA, Oskar S, et al (2014). The effect of metformin on breast cancer outcomes in patients with type 2 diabetes. Cancer Med, 3, 1025-34.

Peairs KS, Barone BB, Snyder CF, et al (2011). Diabetes mellitus and breast cancer outcomes: a systematic review and meta-analysis. J Clin Oncol, 29, 40-6.

Ramjeesingh R, Orr C, Bricks CS, et al (2016). A retrospective study on the role of diabetes and metformin in colorectal cancer disease survival. Curr Oncol, 23, 7.

Sadighi S, Amanpour S, Behrouzi B, et al (2014). Lack of metformin effects on different molecular subtypes of breast cancer under normoglycemic conditions: An in vitro Study. Asian Pac J Cancer Prev, 15, 2287-90.

Sadighi S, Saberian M, Nagafi M, et al (2016). Metformin antiproliferative effect on a cohort of non-diabetic breast cancer patients. Ann Oncol, 27.

Siegel RL, Miller KD, Jemal A (2019). Cancer statistics, 2019. CA Cancer J Clin, 69, 7-34.

Vazquez-Martin A, Oliveras-Ferraros C, Del Barco S, et al (2011). The anti-diabetic drug metformin suppresses self-renewal and proliferation of trastuzumab-resistant tumor-initiating breast cancer stem cells. Breast Cancer Res Treat, 126, 355-64.

Xu H, Chen K, Jia X, et al (2015). Metformin use is associated with better survival of breast cancer patients with diabetes: A Meta-Analysis. Oncologist, 20, 1236-44.

Xu T, Li D, He Y, et al (2018). Prognostic value of metformin for non-small cell lung cancer patients with diabetes. World $J$ Surg Oncol, 16, 60.

Yu H, Zhong X, Gao P, et al (2019). The potential effect of metformin on cancer: An Umbrella Review. Front Endocrinol, $\mathbf{1 0 .}$

Zakikhani M, Dowling R, Fantus IG, et al (2006). Metformin is an AMP kinase-dependent growth inhibitor for breast cancer cells. Cancer Res, 66, 10269-73.

Zhang J, Wu J, He Q, et al (2018). The prognostic value of metformin for advanced non-small cell lung cancer: a systematic review and meta-analysis. Transl Lung Cancer Res, 7, 389-96.

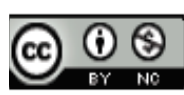

This work is licensed under a Creative Commons AttributionNon Commercial 4.0 International License. 